\title{
Unilateral versus Bilateral Groin Puncture for Atrial Fibrillation Ablation: Multi-Center Prospective Randomized Study
}

\author{
Hee Tae Yu* ${ }^{1 *}$, Dong Geum Shin ${ }^{2 *}$, Jaemin Shim³ , Gi-Byoung Nam, Won Woo Yoo ${ }^{1}$, Ji Hyun Lee ${ }^{4}$, \\ Tae-Hoon Kim ${ }^{1}$, Jae-Sun Uhm ${ }^{1}$, Boyoung Joung ${ }^{1}$, Moon-Hyoung Lee ${ }^{1}$, Young-Hoon Kim ${ }^{3}$, and Hui-Nam Pak ${ }^{1}$ \\ ${ }^{1}$ Department of Cardiology, Yonsei University Health System, Seoul; \\ ${ }^{2}$ Department of Cardiology, Hallym University Kangnam Sacred Heart Hospital, Seoul; \\ ${ }^{3}$ Department of Cardiology, Korea University Cardiovascular Center, Seoul; \\ ${ }^{4}$ Department of Cardiology, Asan Medical Center, Ulsan University, Seoul, Korea.
}

\begin{abstract}
Purpose: Catheter ablation for atrial fibrillation (AF) requires heavy anticoagulation and uncomfortable post-procedural hemostasis. We compared patient satisfaction with and the safety of unilateral groin (UG) puncture-single trans-septal (ST) ablation with conventional bilateral groin (BG) puncture-double trans-septal (DT) ablation in paroxysmal AF patients.

Materials and Methods: We enrolled 222 patients with paroxysmal AF (59.4 \pm 10.7 years old) who were randomized in a 2:1 manner into UG-ST ablation ( $\mathrm{n}=148$ ) and BG-DT ablation $(\mathrm{n}=74)$ groups. If circumferential pulmonary vein isolation could not be achieved after three attempts of touch-up ablation in the UG-ST group, the patient was crossed over to BG-DT by performing a left groin puncture.

Results: Ten patients in the UG-ST group (6.8\%) required crossover to the BG-DT approach. There were no significant differences in procedure time ( $p=0.144)$ and major complications rate $(p>0.999)$ between the UG-ST and BG-DT groups. Access site pain ( $p=0.014)$, back pain $(p=0.023)$, and total pain $(p=0.015)$ scores were significantly lower for the UG-ST than BG-DT group as assessed by the Visual Analog Scale. Over 20.2 \pm 8.7 months of follow up, there was no difference in AF recurrence free-survival rates between the two groups (Log rank, $p=0.984$ ).

Conclusion: UG-ST AF ablation is feasible and safe, and was found to significantly reduce post-procedural hemostasis-related discomfort, compared to the conventional DT approach, in patients with paroxysmal AF.
\end{abstract}

Key Words: Atrial fibrillation, catheter ablation, groin, puncture

\section{INTRODUCTION}

Radiofrequency catheter ablation (RFCA) has been shown to effectively reduce the burden of atrial fibrillation (AF). ${ }^{1,2}$ Since

Received: November 6, 2018 Revised: February 7, 2019

Accepted: February 12, 2019

Corresponding author: Hui-Nam Pak, MD, PhD, Department of Cardiology, Yonsei University Health System, 50-1 Yonsei-ro, Seodaemun-gu, Seoul 03722, Korea. Tel: 82-2-2228-8459, Fax: 82-2-2227-7732, E-mail: hnpak@yuhs.ac

*Hee Tae Yu and Dong Geum Shin contributed equally to this work.

-The authors have no potential conflicts of interest to disclose.

(C) Copyright: Yonsei University College of Medicine 2019

This is an Open Access article distributed under the terms of the Creative Commons Attribution Non-Commercial License (https://creativecommons.org/licenses/ by-nc/4.0) which permits unrestricted non-commercial use, distribution, and reproduction in any medium, provided the original work is properly cited.
Haïssaguerre, et al. ${ }^{3}$ reported focal ablation of pulmonary vein (PV) triggers by single ablation catheter after single trans-septal puncture in 1998, the indications for RFCA have been expanded in light of accumulating clinical experience and evidence. ${ }^{4}$ Circumferential pulmonary vein isolation (CPVI) remains the cornerstone of AF ablation, ${ }^{5,6}$ and current technologies, such as irrigated tip ablation catheters, ${ }^{7}$ contact force technology, ${ }^{8}$ and three-dimensional (3D) electroanatomical mapping with software monitoring for catheter stability, ${ }^{9}$ have allowed for efficient and long-lasting CPVI, as well as improved rhythm outcomes, in AF catheter ablation. For CPVI, most operators place two long sheaths across the interatrial septum, one for a PV mapping catheter and the other for the ablation catheter. In this double trans-septal (DT) method, CPVI is performed while watching PV potentials. This technique requires 
bilateral groin (BG) puncture and a relatively long post-procedural hemostasis time, with both lower extremities remaining extended after the procedure. Indeed, the long duration of post-procedural immobilization for hemostasis can cause back pain, discomfort, negative immunomodulatory effects, ${ }^{10}$ and bleeding complications. In addition, Asian patients are known to be vulnerable to bleeding under anticoagulation. ${ }^{11}$ Recently, commercialized cryoballoon PV ablation was found to result in clinical outcomes comparable to RFCA by a unilateral groin (UG) puncture, ${ }^{12}$ and the invasiveness of the procedure became an important issue. In the present study, we hypothesized that UG puncture-single trans-septal (ST) CPVI would be feasible and safe and would reduce patient postprocedural discomfort during hemostasis after RFCA for paroxysmal AF, as the patient has one leg that is free to move after the UG-ST procedure, compared to the BG-DT approach. The purpose of this study was to compare rates of procedural success and complications, as well as post-procedural patient satisfaction and rhythm outcomes, between UG-ST and BG-DT CPVIs via a multi-center prospective randomized trial design.

\section{MATERIALS AND METHODS}

\section{Study population}

The study protocol was approved by the institutional review boards of the participating hospitals (ClinicalTrials.gov Identifier: NCT02438176). The study received ethics committee approval from each hospital [The Institutional Review Board of Severance Cardiovascular Hospital (IRB: 4-2015-0170), The Institutional Review Board of Korea University Cardiovascular Center, The Institutional Review Board of Asan Medical Center] and complied with the Declaration of Helsinki. All participants provided written informed consent. We originally planned to recruit 70 patients in the UG-ST group and 35 patients in the
BG-DT group, however, we recruited additional participants to further improve the statistical power of the study under the approval of the ethics committee. Finally, from April 2015 to June 2017, a total of 222 patients with anti-arrhythmic drug (AAD) refractory symptomatic paroxysmal AF were enrolled in this prospective, randomized, single blind study performed at three tertiary hospitals in Seoul, Korea. Paroxysmal AF was defined as a history of $\geq$ one episode of self-terminating AF lasting $\leq 7$ days. Exclusion criteria consisted of not agreeing to enroll in the study, persistent AF, difficulty in performing a single groin puncture due to vascular disease or venous anomaly, history of previous RFCA or cardiac surgery, and valvular or structural heart disease other than left ventricular hypertrophy. Patients were randomly assigned to the UG-ST or BG-DT groups using a table of random numbers in a 2:1 ratio (Fig. 1). In the case of failed PV isolation despite three attempts at touch-up ablation in the UG-ST group, the patient was crossed over to BG-DT. All AADs were withheld for $>5$ half-lives before the procedure. All patients received at least 3 weeks of non-vitamin K antagonist oral anticoagulant (NOAC) prior to the procedure. ${ }^{13}$ Prior to ablation, all patients underwent 64-channel multi-detector computed tomography (CT), and pre-procedural transesophageal echocardiogram was evaluated in patients with $\mathrm{CHA}_{2} \mathrm{DS}_{2}$-VASc scores $\geq 2$, suspicious left atrial appendage thrombus on CT, or potential for poor adherence to anticoagulation therapy.

\section{Groin puncture and catheter setting}

Procedures were performed under conscious sedation/analgesia. For the BG-DT group (Fig. 2A), a 14-Fr venous tripod sheath was introduced to the left femoral vein (for duodecapolar, decapolar, and quardripolar catheters), and a 6-Fr arterial locking sheath was introduced into the left femoral artery (for reference catheter and blood pressure monitoring), respectively. Two long trans-septal sheaths (Schwartz left-1,

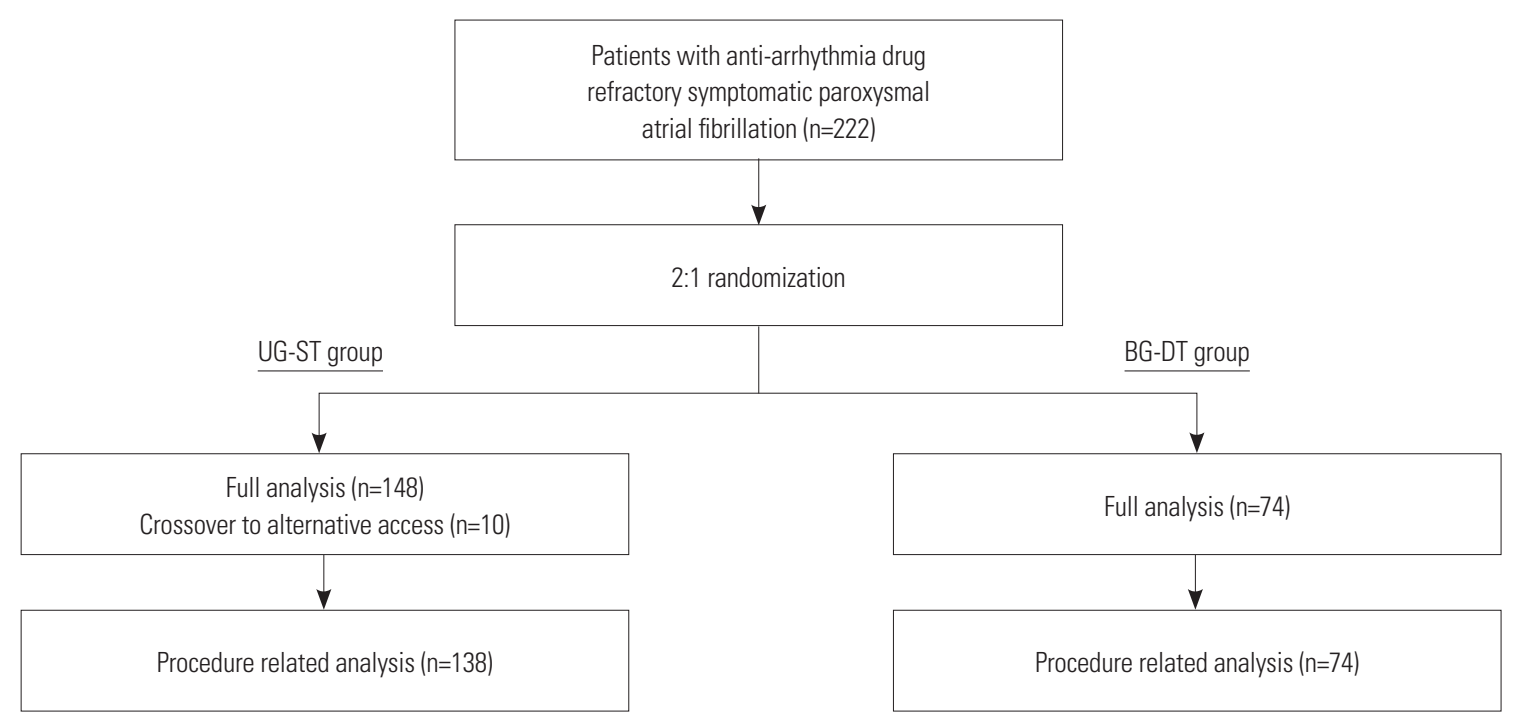

Fig. 1. Flow diagram of study population enrollment. UG-ST, unilateral groin puncture-single trans-septal; BG-DT, bilateral groin puncture-double trans-septal. 

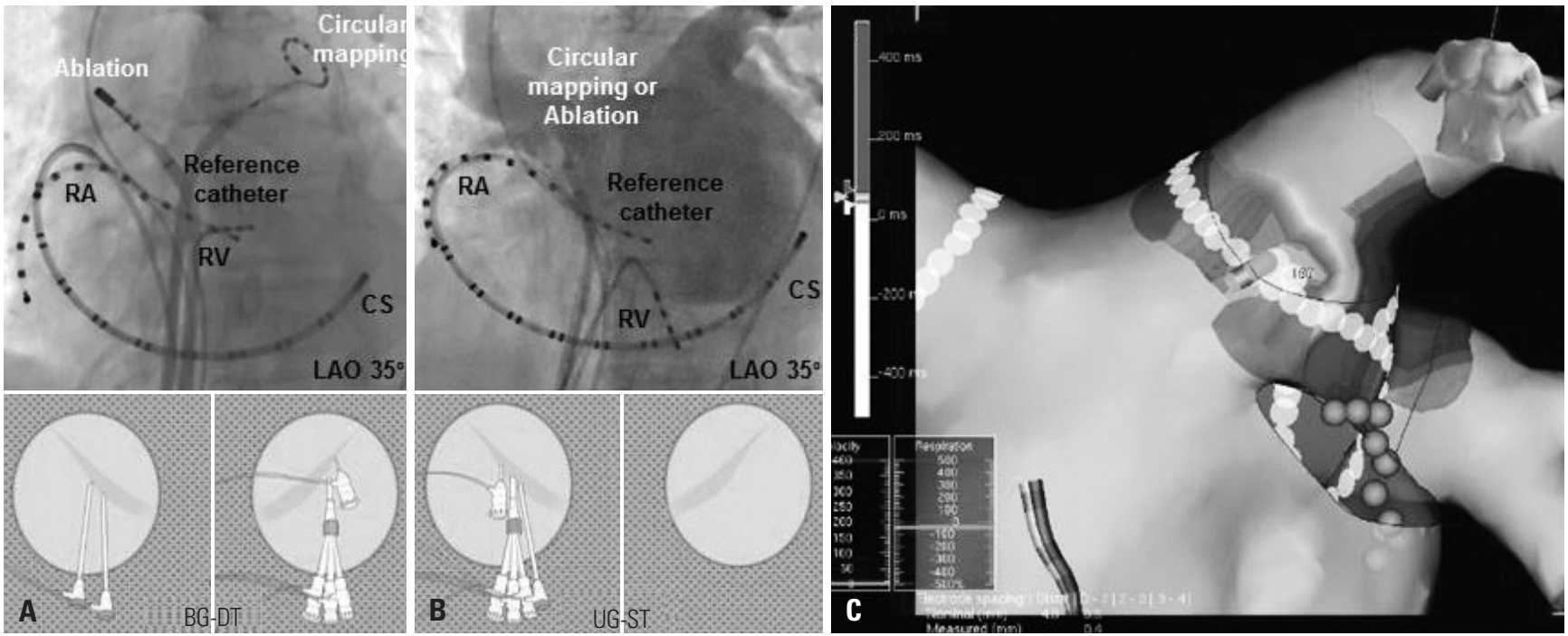

Fig. 2. Representative images of conventional bilateral groin puncture-double trans-septal ablation (BG-DT) (A) and unilateral groin puncture-single trans-septal ablation (UG-ST) (B) strategies. If there was a remnant PV potential after anatomical and local potential guided CPVI in the UG-ST group, we mapped and marked the potential PV conduction site on a three-dimensional electroanatomical map and added tough-up ablation after an additional exchange of ablation and mapping catheters (C). CPVI, circumferential pulmonary vein isolation; PV, pulmonary vein; $R A$, right atrium; $R V$, right ventricle; $C S$, coronary sinus; LAO, left anterior oblique.

braided, 8-Fr, Abbott Inc., Minnetonka, MN, USA) were then introduced into the right femoral vein (for an ablation catheter and a PV mapping catheter).

In the UG-ST group, we placed a 14-Fr tripod sheath and a trans-septal long sheath into the right femoral vein, and a 6-Fr artery sheath into the right femoral artery; there was no left side groin access (Fig. 2B). We introduced the same catheters used in the BG-DT group through three vascular sheaths; however, the ablation and PV mapping catheters shared a long sheath.

\section{Electrophysiological mapping and ablation}

Intracardiac electrograms were recorded using the Prucka CardioLab $^{\text {TM }}$ Electrophysiology system (General Electric Medical Systems, Inc., Milwaukee, WI, USA). RFCA was performed in all patients using 3D electroanatomical mapping (NavX, Abbott Inc.) merged with 3D spiral CT. After obtaining multiview pulmonary venograms and securing trans-septal access, the $3 \mathrm{D}$ geometries of both the LA and PV were obtained using a 3D-electroanatomical mapping system (NavX, Abbott Inc.), which were then merged with 3D spiral CT images. Systemic anticoagulation was performed with intravenous heparin to maintain an activated clotting time of 350-400 seconds during the procedure. Details on the RFCA technique and the strategy used in the current study have been described previously by our group. ${ }^{14}$ Briefly, an open irrigated-tip catheter (Coolflex, Abbott Inc.; Thermocool, Johnson \& Johnson Inc., Diamond Bar, CA, USA; 25-35 W; irrigation rate of 10-15 mL/ min, continuous and endurance ablation with staying $30 \mathrm{sec}-$ onds in each ablation point) was used to deliver RF energy for ablation. All patients underwent CPVI and cavo-tricuspid isthmus (CTI) ablation. Successful CPVI was defined as elimi- nation of all PV potentials along the antrum and inside the vein. Bidirectional CTI block was confirmed by differential pacing.

In the UG-ST group, we exchanged he ablation catheter with a PV mapping catheter after circumferential PV ablation, localized the remaining entrance site of PV potentials with quick 3D mapping (NavX, Abbott Inc.) (Fig. 2C), and achieved $\mathrm{PV}$ isolation by touch-up ablation. If we failed to isolate four PVs after three attempts at repeated touch-up ablation, we conducted left groin puncture and additional trans-septal puncture, and isolated four PVs during simultaneous PV potential monitoring by PV mapping catheter (cross over to the BG-DT protocol). As a final step, mappable AF triggers or atrial premature beats seen with isoproterenol infusion $(5 \mu \mathrm{g} / \mathrm{min})$ were carefully mapped and the corresponding non-PV foci were ablated as much as possible. ${ }^{15}$

\section{Hemostasis and questionnaire}

After sheath removal, manual compression was applied to the puncture sites until hemostasis was achieved. After 4 hours of immobilization, the puncture sites were inspected. In the BGDT group, patients were asked not to flex either lower extremity, and immobilization was maintained overnight. In contrast, patients in the UG-ST group were allowed to move their left leg (opposite to the groin puncture side) and short distance ambulation after 6 hours of stabilization. Major hematoma was defined as bleeding or a significantly visible hematoma of the groin site when the hospital discharge was delayed by more than one day. A NOAC was restarted on the day of the procedure after confirming hemostasis. Oral anticoagulation was continued for at least 2 months after the procedure in all patients.

A questionnaire covering subjective discomfort during he- 
mostasis was provided to patients one day after the procedure, prior to hospital discharge, with standardized instructions in accordance with previously published guidelines. ${ }^{16}$ The questionnaire, which was completed by the patients, consisted of a visual analog scale (VAS) of 0 to 10 covering overall discomfort, access site pain, back pain, ability to use the restroom, ability to eat and perform self-care, and walking (Supplementary Fig. 1 , only online). The total VAS score was calculated by summation of VAS scores from each category.

\section{Follow-up after procedure}

According to standard RFCA procedures, AADs were discontinued in the majority of patients, but continued in $6.8 \%(15 / 222)$ of patients with a high chance of early recurrence or symptomatic atrial arrhythmias at discharge. All patients were scheduled for outpatient clinic follow up at 1, 3, 6, and 12 months, and every 6 months thereafter or upon becoming symptomatic. Rhythm follow ups were performed at every visit by ECG, and a 24-hour Holter monitoring and/or event recording was conducted at 3- and 6-month intervals during the first year and every six months thereafter according to the 2012 Heart Rhythm Society/European Heart Rhythm Society/European Cardiac Arrhythmia Society Expert Consensus Statement guidelines. ${ }^{17}$ Additionally, if a patient presented with palpitations, Holter monitoring or event recording was used to screen for arrhythmias. We defined recurrence of $\mathrm{AF}$ as any episode of $\mathrm{AF}$ or atrial tachycardia lasting longer than 30 seconds. Early recurrence was defined as any episode of recurrent AF occurring within 3 months of the blanking period. Any ECG documentation of AF recurrence after the blanking period was diagnosed as clinical recurrence. The primary outcomes consisted of CPVI success rate, procedure time, and peri-procedural complication rates within 30 days of the procedure, including groin site complications. The secondary outcomes were VAS score for discomfort during hemostasis and clinical recurrence rates of atrial arrhythmias.

\section{Statistical analysis}

We established treatment superiority based on VAS according to a previous patient satisfaction study. Specifically, a decrease in VAS score $>33 \%$ was considered to represent an increase in patient satisfaction. ${ }^{18}$ A statistical power of $80 \%$ and a twotailed type 1 error of $5 \%$ was used to identify significant differences, considering a potential dropout rate of $10 \%$. Patients were assigned in a 2:1 ratio out of consideration for the possibility of procedure crossover, such as from a UG to bilateral approach, and a total of 222 patients was enrolled in the study (UG-ST group 148 patients and BG-DT group 74 patients). Continuous variables are reported as mean \pm standard deviation and were analyzed using Student's t-test or the MannWhitney U test as appropriate. Normality was determined using the Kolmogorov-Smirnov goodness-of-fit test. Categorical variables are reported as counts and proportions, and were an- alyzed using Pearson's chi-square test or Fisher's exact test as appropriate. Kaplan-Meier analyses with log rank tests were used to compare AF recurrence-free survival over time between the two groups. Statistical analysis was performed using SPSS (version 23.0, IBM Corp., Armonk, NY, USA) software for Windows. A $p$-value $<0.05$ was considered statistically significant.

\section{RESULTS}

\section{Baseline characteristics}

A total of 222 patients were enrolled in the study and were randomized to the UG-ST group and BG-DT groups in 2:1 manner, with 148 patients in the UG-ST group and 74 patients in the in the BG-DT group. The mean patient age was $59.4 \pm 10.7$ years, and $154(69.4 \%)$ patients were male. The mean $\mathrm{CHA}_{2} \mathrm{DS}_{2}$-VASc score was $1.8 \pm 1.6$. The two groups were balanced in regards to baseline characteristics after randomization as shown in Table 1.

\section{Acute procedural outcomes}

Bidirectional block of CPVI and CTI ablation was successfully achieved in all patients of both groups. Among the 148 patients in the UG-ST group, 10 (6.8\%) required procedure crossover with additional left groin puncture and trans-septal puncture due to difficulty in achieving CPVI after three trials of touch-up

Table 1. Baseline Clinical Characteristics

\begin{tabular}{lcccc}
\hline & $\begin{array}{c}\text { Overall } \\
\text { (n=222) }\end{array}$ & $\begin{array}{c}\text { UG-ST } \\
\text { group } \\
(\mathbf{n = 1 4 8})\end{array}$ & $\begin{array}{c}\text { BG-DT } \\
\text { group } \\
\text { (n=74) }\end{array}$ & p value \\
\hline Age (yr) & $59.4 \pm 10.7$ & $58.7 \pm 11.0$ & $60.9 \pm 10.0$ & 0.149 \\
Male sex & $154(69.4)$ & $108(73.0)$ & $46(62.2)$ & 0.100 \\
\hline BMI (kg/m²) & $25.0 \pm 3.3$ & $25.1 \pm 3.4$ & $24.7 \pm 3.1$ & 0.383 \\
Comorbidities & & & & \\
\hline Heart failure & $18(8.1)$ & $12(8.1)$ & $6(8.1)$ & $>0.999$ \\
\hline Hypertension & $101(45.5)$ & $66(44.6)$ & $35(47.3)$ & 0.703 \\
\hline Diabetes mellitus & $41(18.5)$ & $25(16.9)$ & $16(21.6)$ & 0.392 \\
\hline Stroke or TIA & $28(12.6)$ & $18(12.2)$ & $10(13.5)$ & 0.775 \\
\hline Vascular disease & $26(11.7)$ & $17(11.5)$ & $9(12.2)$ & 0.883 \\
\hline CHA ${ }_{2}$ DS ${ }_{2}$-VASc score & $1.8 \pm 1.6$ & $1.7 \pm 1.6$ & $1.9 \pm 1.5$ & 0.381 \\
\hline Echocardiography & & & & \\
\hline LA diameter (mm) & $39.5 \pm 5.7$ & $39.7 \pm 6.1$ & $39.2 \pm 4.8$ & 0.543 \\
\hline LAVI (mL/m $\left.{ }^{2}\right)$ & $34.7 \pm 12.5$ & $35.3 \pm 13.5$ & $33.6 \pm 10.3$ & 0.357 \\
\hline LVEF (\%) & $64.7 \pm 7.3$ & $64.2 \pm 7.7$ & $65.6 \pm 6.4$ & 0.166 \\
\hline E/Em & $9.9 \pm 3.9$ & $10.1 \pm 4.0$ & $9.6 \pm 3.5$ & 0.370 \\
\hline LVEDD (mm) & $49.7 \pm 4.0$ & $49.8 \pm 4.2$ & $49.6 \pm 3.6$ & 0.810 \\
\hline UGST & & & \\
\hline
\end{tabular}

UG-ST, unilateral groin puncture-single trans-septal; BG-DT, bilateral groin puncture-double trans-septal; BMI, body mass index; TIA, transient ischemic attack; LA, left atrium; LAVI, LA volume index; LVEF, LV ejection fraction; E/Em, early mitral inflow velocity to early diastolic mitral annular velocity ratio; LVEDD, left ventricular end diastolic dimension.

Variables are presented as mean \pm standard deviation or count (percentage). 
ablation. The 10 crossover subjects were all male, had no comorbidities other than hypertension, and had lower $\mathrm{CHA}_{2} \mathrm{DS}_{2}$ VASc score $(0.9 \pm 0.7$ vs. $1.8 \pm 1.7, p=0.005)$ than the remainder of patients in the UG-ST group (Supplementary Table 1, only online). Their other characteristics, such as echocardiographic parameters or procedure time, ablation time, and fluoroscopic time, were not significantly different from the remainder of patients in the UG-ST group. Table 2 summarizes the acute procedural outcomes of this study. Total procedure time ( $p=0.144)$, ablation time ( $p=0.248)$, and fluoroscopic time ( $p=$ 0.717 ) were not significantly different between the two groups. The overall procedure-related complication rate was $5.4 \%$ $(12 / 222)$. There were no significant differences in major complication rate $(2.0 \%$ vs. $2.7 \%, p>0.999)$, including cardiac tamponade ( $1.4 \%$ vs. $2.7 \%, p=0.602)$ and atrioesophageal fistula $(0.7 \%$ vs. $0 \%, p>0.999)$, between the two groups. Likewise, there were no instances of stroke or transient ischemic attack after RFCA in either group. Minor vascular complications, including femoral arteriovenous fistula not requiring surgical treat- ment or transfusion, and thigh hematoma occurred less frequently in the UG-ST group than in the BG-DT group (1.4\% vs. $6.8 \%, p=0.043$ ). In addition, after excluding the 10 patients in the UG-ST group who were crossed-over to the double transseptal puncture, acute procedural outcome and major complication rates were consistent with those in the BG-DT group (Supplementary Table 1, only online). There were no procedure-related complications in the 10 crossover patients either.

\section{Post-procedural discomfort during hemostasis}

A questionnaire for post-procedural discomfort during hemostasis was successfully administered according to guidelines in $97.8 \%(135 / 138)$ of patients in the UG-ST group after excluding the 10 crossover patients and in $98.6 \%$ (73 of 74) patients in the BG-DT group. According to the patient satisfaction survey, total VAS score $(p=0.015)$, access site pain score ( $p=$ $0.014)$, and back pain score $(p=0.023)$ were significantly lower in the UG-ST group than in the BG-DT group (Fig. 3).

Table 2. Procedure Related Characteristics

\begin{tabular}{|c|c|c|c|c|}
\hline & Overall ( $n=222)$ & UG-ST group ( $n=148$ ) & BG-DT group (n=74) & $p$ value \\
\hline Procedure time (min) & $184.0 \pm 37.2$ & $186.6 \pm 37.0$ & $178.8 \pm 37.3$ & 0.144 \\
\hline Ablation time (sec) & $4722.3 \pm 936.2$ & $4773.8 \pm 938.8$ & $4619.4 \pm 928.8$ & 0.248 \\
\hline Fluoroscopy time (min) & $34.8 \pm 12.3$ & $34.6 \pm 12.2$ & $35.2 \pm 12.5$ & 0.717 \\
\hline Major complications & $5(2.3)$ & $3(2.0)$ & $2(2.7)$ & $>0.999$ \\
\hline Cardiac tamponade & $4(1.8)$ & $2(1.4)$ & $2(2.7)$ & 0.602 \\
\hline AE fistula & $1(0.5)$ & $1(0.7)$ & $0(0)$ & $>0.999$ \\
\hline Stroke or TIA & $0(0)$ & $0(0)$ & $0(0)$ & $>0.999$ \\
\hline Minor vascular complications & $7(3.2)$ & $2(1.4)$ & $5(6.8)$ & 0.043 \\
\hline Femoral AV fistula & $3(1.4)$ & $1(0.7)$ & $2(2.7)$ & 0.258 \\
\hline Femoral pseudoaneurysm & $0(0)$ & $0(0)$ & $0(0)$ & $>0.999$ \\
\hline Thigh hematoma & $4(1.8)$ & $1(0.7)$ & $3(4.1)$ & 0.109 \\
\hline
\end{tabular}

UG-ST, unilateral groin puncture-single trans-septal; BG-DT, bilateral groin puncture-double trans-septal; AE, atrioesophageal; TIA, transient ischemic attack; AV, arteriovenous.

Variables are presented as mean \pm standard deviation or count (percentage).
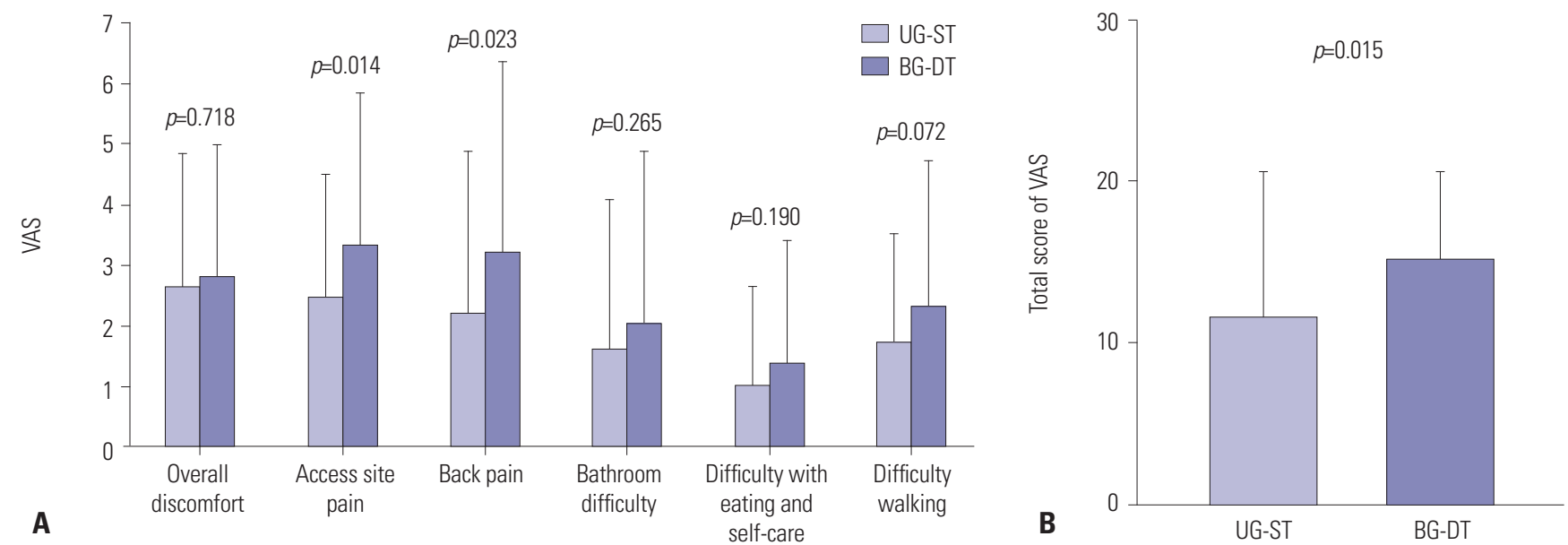

Fig. 3. Comparison of questionnaire results for post-procedural discomfort during hemostasis between the two groups. (A) Each component of quality of life was measured using a 0-10 VAS. (B) Total score of VAS results. UG-ST, unilateral groin puncture-single trans-septal; BG-DT, bilateral groin puncture-double trans-septal; VAS, visual analog scale. 


\section{Clinical rhythm outcomes}

Table 3 shows the clinical rhythm outcomes of the study groups. During 20.2 \pm 8.7 months of follow up, there was clinical recurrence in 16 patients in the UG-ST group and 8 patients in the BG-DT group ( $10.8 \%$ vs. $10.8 \%, p>0.999)$. After excluding the 10 crossover patients of the UG-ST group, the rate of clinical recurrence was $10.9 \%(15 / 138)$. The rate of early recurrence was not different between the UG-ST and BG-DT groups ( $p=0.541$ ). Overall, Kaplan-Meier analyses showed comparable rhythm outcomes in the UG-ST group, compared to the BG-DT group (Log rank $p=0.984$ ) (Fig. $4 \mathrm{~A}$ ), as well as after excluding patients being treated with an $\mathrm{AAD}(\log \operatorname{rank} p=0.976)$ (Fig. 4B).

\section{DISCUSSION}

In this multicenter prospective randomized study for comparison of a unilateral groin puncture procedure with that of conventional bilateral groin puncture, UG-ST was a feasible and safe ablation strategy for CPVI, even though it was a single trans-septal procedure. Moreover, the UG-ST method reduced post-procedural discomfort significantly during hemostasis, and the rate of clinical recurrence of AF was comparable to

Table 3. Clinical Rhythm Outcomes

\begin{tabular}{lcccc}
\hline & $\begin{array}{c}\text { Overall } \\
(\mathbf{n = 2 2 2})\end{array}$ & $\begin{array}{c}\text { UG-ST } \\
\text { group } \\
(\mathbf{n = 1 4 8})\end{array}$ & $\begin{array}{c}\text { BG-DT } \\
\text { group } \\
(\mathbf{n = 7 4 )}\end{array}$ & $\boldsymbol{p}$ value \\
\hline $\begin{array}{l}\text { Post ablation AAD use at } \\
\quad \text { months }\end{array}$ & $15(6.8)$ & $8(5.4)$ & $7(9.5)$ & 0.257 \\
Follow up (month) & $20.2 \pm 8.7$ & $20.4 \pm 9.0$ & $19.6 \pm 8.1$ & 0.481 \\
\hline Early recurrence & $41(18.5)$ & $29(19.6)$ & $12(16.2)$ & 0.541 \\
Clinical recurrence & $24(10.8)$ & $16(10.8)$ & $8(10.8)$ & $>0.999$ \\
\hline
\end{tabular}

UG-ST, unilateral groin puncture-single trans-septal; BG-DT, bilateral groin puncture-double trans-septal; $A A D$, anti-arrhythmic drug.

Variables are presented as mean \pm standard deviation or count (percentage).

A

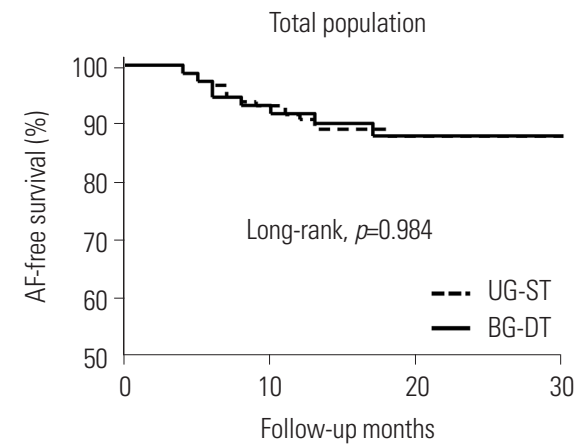

Number at risk

$$
\text { UG-ST }
$$

BG-DT

$$
148
$$$$
123
$$$$
62
$$

that of conventional bilateral groin puncture with the double trans-septal method (BG-DT group).

There is a general consensus that CPVI is the cornerstone of $\mathrm{AF}$ catheter ablation in both paroxysmal $\mathrm{AF}$ and persistent $\mathrm{AF}^{2,19}$ Although a reconnected $\mathrm{PV}$ potential is known to be the main mechanism of AF recurrence, ${ }^{14}$ achieving long-term PV isolation has increased significantly with technological advances in ablation catheters, mapping, and imaging technology. ${ }^{7-9}$ The central hypothesis of this study was that successful CPVI could be achieved by a sophisticated ST-single catheter antral ablation without continuous PV potential monitoring by a PV mapping catheter, which requires DT access. While it is inconvenient for operators to switch between the ablation catheter and PV mapping catheter through a single trans-septal sheath, UG-ST ablation successfully achieved CPVI in the majority of patients (93.2\%) without worsening of procedure times, complication rates, or clinical outcomes.

AF ablation is a complex procedure that requires multiple catheter mappings, a relatively long procedure time, intra-procedural heavy anticoagulation, and uninterrupted peri-procedural anticoagulation. ${ }^{20}$ Therefore, complications associated with vascular access are relatively common: groin hematoma, retroperitoneal hematoma, femoral pseudoaneurysm, femoral arteriovenous fistula, and hemo- or pneumothorax..$^{21-23}$ To reduce the number of vascular access sites, such as subclavian or internal jugular venous access, we have been using a bilateral groin puncture method. ${ }^{24,25}$ In this study, we further simplified vascular access in the UG-ST method, similar to the generalized setting when performing cryoballoon ablation. ${ }^{26}$ The UG-ST method significantly reduced patient discomfort during hemostasis, because it allowed the patients to move their left side lower extremity.

There has been only one study to date that has reported on patient satisfaction during and immediately after AF ablation procedures, and dissatisfaction mainly occurs due to excess pain during and after ablation. ${ }^{27}$ Taking into account both the procedure time and hemostatic time, patients undergoing $\mathrm{AF}$

B

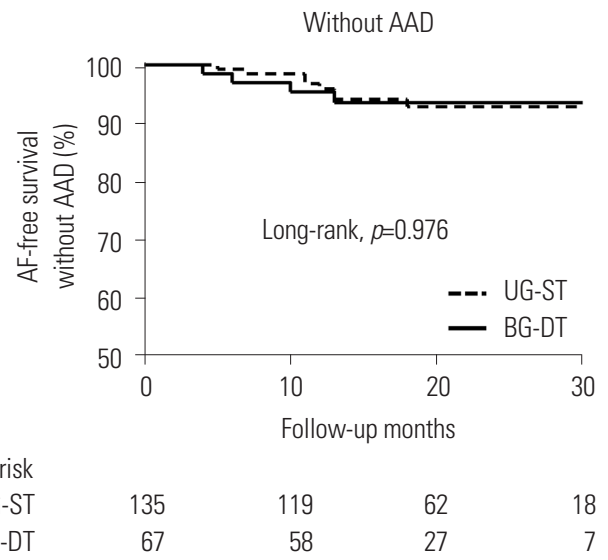

Fig. 4. Kaplan-Meier analysis of $A F$ recurrence-free survival $(A)$ and $A F$ recurrence-free survival off- $A A D(B)$ after catheter ablation between the two groups. $A F$, atrial fibrillation; $A A D$, anti-arrhythmic drug; UG-ST, unilateral groin puncture-single trans-septal; $B G-D T$, bilateral groin puncture-double trans-septal. 
catheter ablation must remain in a supine position for at least 8 hours, which is both uncomfortable and difficult, especially in older adult patients with back pain. We proved that the UGST method is valuable to improve satisfaction of patients who undergo AF catheter ablation in this study.

Although PV stenosis is uncommon after antral CPVI, it can occur after deeper and massive ablations. ${ }^{28}$ In cases of a remnant PV potential after initial CPVI by SG-ST single catheter ablation, we strongly recommend the use of quick 3D-activation mapping, as shown in Fig. 2C, before touch-up ablation. This method is not only very efficient to eliminate PV potential with minimal RF energy delivery, but also effective in preventing the temptation to ablate in PV inside. In order to avoid this complication, patients being treated by the UG-ST method were crossed over to the BG-DT method, if three touch-up ablations failed to achieve secure CPVI. Another potential complication of the UG-ST method is air-embolism during repeated exchange of the ablation catheter and PV mapping catheter. ${ }^{29}$ Although none of the patients in this study had any embolic events, gentle catheter removal from a trans-septal sheath and repeated saline flushing at every catheter exchange is strongly recommended. Although the UG-ST method is limited with respect to the lack of differential pacing to confirm bidirectional blocks after LA linear ablation, it may also be utilized for complex fractionated atrial electrogram-guided ablation and rotor ablation in patients with persistent $\mathrm{AF}$.

Several limitations of the present study should be considered. First, because the study questionnaire was designed to evaluate discomfort within 24 hours of the $\mathrm{AF}$ ablation procedure, we did not assess pain after discharge. Second, rates of groin complications may also be affected by the choice of NOAC. However, we found no significant difference in the proportion of commercially available NOACs between two groups ( $p=$ 0.433 ), and both groups were managed with the same activated clotting time target and post-procedural protamine delivery. Third, the two approaches for groin/transseptal puncture applied in this study differed with respect to two variables: single versus double groin puncture and single versus double transseptal puncture.

In conclusion, UG-ST is a feasible and safe ablation strategy for CPVI despite only using a single trans-septal procedure. Moreover, the UG-ST method reduces post-procedural discomfort significantly during hemostasis, with a rate of clinical AF recurrence comparable to that of the conventional BG-DT method.

\section{ACKNOWLEDGEMENTS}

This research was supported by a grant (A085136) from the Korea Health 21 R\&D Project, Ministry of Health and Welfare and a grant (NRF-2017R1A2B4003983; 2017R1C1B1008292) from the Basic Science Research Program run by the National Research Foundation of Korea (NRF).

\section{AUTHOR CONTRIBUTIONS}

Conceptualization: Hee Tae Yu, Dong Geum Shin, Hui-Nam Pak. Data curation: Young-Hoon Kim, Gi-Byoung Nam, Hui-Nam Pak. Formal analysis: Hee Tae Yu, Dong Geum Shin. Funding acquisition: Hui-Nam Pak. Investigation: Hee Tae Yu, Dong Geum Shin, Jaemin Shim, Won Woo Yoo, Ji Hyun Lee, Tae-Hoon Kim, Jae-Sun Uhm, Boyoung Joung, Moon-Hyoung Lee. Methodology: Hee Tae Yu, Dong Geum Shin, Hui-Nam Pak. Project administration: Hui-Nam Pak. Resources: Young-Hoon Kim, Gi-Byoung Nam, Hui-Nam Pak. Software: Hui-Nam Pak. Supervision: Young-Hoon Kim, Gi-Byoung Nam, Moon-Hyoung Lee, Hui-Nam Pak. Validation: Young-Hoon Kim, GiByoung Nam. Visualization: Hee Tae Yu, Dong Geum Shin, Hui-Nam Pak. Writing_original draft: Hee Tae Yu, Dong Geum Shin, Hui-Nam Pak. Writing_review \& editing: Hee Tae Yu, Hui-Nam Pak.

\section{ORCID iDs}

Hee Tae Yu https://orcid.org/0000-0002-6835-4759

Dong Geum Shin https://orcid.org/0000-0002-5473-9068 Jaemin Shim https://orcid.org/0000-0001-8251-1522 Gi-Byoung Nam https://orcid.org/0000-0003-4391-5406 Won Woo Yoo https://orcid.org/0000-0002-1884-8224 Ji Hyun Lee https://orcid.org/0000-0002-7162-1248 Tae-Hoon Kim https://orcid.org/0000-0003-4200-3456 Jae-Sun Uhm https://orcid.org/0000-0002-1611-8172 Boyoung Joung https://orcid.org/0000-0001-9036-7225 Moon-Hyoung Lee https://orcid.org/0000-0002-7268-0741 Young-Hoon Kim https://orcid.org/0000-0002-4254-647X Hui-Nam Pak https://orcid.org/0000-0002-3256-3620

\section{REFERENCES}

1. Wilber DJ, Pappone C, Neuzil P, De Paola A, Marchlinski F, Natale A, et al. Comparison of antiarrhythmic drug therapy and radiofrequency catheter ablation in patients with paroxysmal atrial fibrillation: a randomized controlled trial. JAMA 2010;303:333-40.

2. Kirchhof P, Benussi S, Kotecha D, Ahlsson A, Atar D, Casadei B, et al. 2016 ESC Guidelines for the management of atrial fibrillation developed in collaboration with EACTS. Eur Heart J 2016;37:2893962.

3. Haïssaguerre M, Jaïs P, Shah DC, Takahashi A, Hocini M, Quiniou $\mathrm{G}$, et al. Spontaneous initiation of atrial fibrillation by ectopic beats originating in the pulmonary veins. N Engl J Med 1998;339:659-66.

4. Wazni OM, Marrouche NF, Martin DO, Verma A, Bhargava M, Saliba W, et al. Radiofrequency ablation vs antiarrhythmic drugs as first-line treatment of symptomatic atrial fibrillation: a randomized trial. JAMA 2005;293:2634-40.

5. Haïssaguerre M, Shah DC, Jaïs P, Hocini M, Yamane T, Deisenhofer I, et al. Electrophysiological breakthroughs from the left atrium to the pulmonary veins. Circulation 2000;102:2463-5.

6. Oral H, Knight BP, Tada H, Ozaydin M, Chugh A, Hassan S, et al. Pulmonary vein isolation for paroxysmal and persistent atrial fibrillation. Circulation 2002;105:1077-81.

7. Hwang ES, Pak HN, Park SW, Park JS, Joung B, Choi D, et al. Risks and benefits of an open irrigation tip catheter in intensive radiofrequency catheter ablation in patients with non-paroxysmal atrial fibrillation. Circ J 2010;74:644-9.

8. Shurrab M, Di Biase L, Briceno DF, Kaoutskaia A, Haj-Yahia S, Newman D, et al. Impact of contact force technology on atrial fibrillation ablation: a meta-analysis. J Am Heart Assoc 2015;4:e002476. 
9. Bertaglia E, Bella PD, Tondo C, Proclemer A, Bottoni N, De Ponti $\mathrm{R}$, et al. Image integration increases efficacy of paroxysmal atrial fibrillation catheter ablation: results from the CartoMerge Italian Registry. Europace 2009;11:1004-10.

10. Radulovic M, Weber C, Spiess J. The effect of acute immobilization stress on the abundance of corticotropin-releasing factor receptor in lymphoid organs. J Neuroimmunol 2000;103:153-64.

11. Lip GY, Wang KL, Chiang CE. Non-vitamin K antagonist oral anticoagulants (NOACs) for stroke prevention in Asian patients with atrial fibrillation: time for a reappraisal. Int J Cardiol 2015;180:24654.

12. Kuck KH, Brugada J, Fürnkranz A, Metzner A, Ouyang F, Chun $\mathrm{KR}$, et al. Cryoballoon or radiofrequency ablation for paroxysmal atrial fibrillation. N Engl J Med 2016;374:2235-45.

13. Shin DG, Kim TH, Uhm JS, Kim JY, Joung B, Lee MH, et al. Early experience of novel oral anticoagulants in catheter ablation for atrial fibrillation: efficacy and safety comparison to warfarin. Yonsei Med J 2016;57:342-9.

14. Kim TH, Park J, Uhm JS, Kim JY, Joung B, Lee MH, et al. Challenging achievement of bidirectional block after linear ablation affects the rhythm outcome in patients with persistent atrial fibrillation. J Am Heart Assoc 2016;5:e003894.

15. Kim IS, Yang PS, Kim TH, Park J, Park JK, Uhm JS, et al. Clinical significance of additional ablation of atrial premature beats after catheter ablation for atrial fibrillation. Yonsei Med J 2016;57:7280.

16. Cooper CJ, El-Shiekh RA, Cohen DJ, Blaesing L, Burket MW, Basu A, et al. Effect of transradial access on quality of life and cost of cardiac catheterization: a randomized comparison. Am Heart J 1999;138(3 Pt 1):430-6.

17. Calkins H, Kuck KH, Cappato R, Brugada J, Camm AJ, Chen SA, et al. 2012 HRS/EHRA/ECAS Expert Consensus Statement on Catheter and Surgical Ablation of Atrial Fibrillation: recommendations for patient selection, procedural techniques, patient management and follow-up, definitions, endpoints, and research trial design. Europace 2012;14:528-606.

18. Jensen MP, Chen C, Brugger AM. Interpretation of visual analog scale ratings and change scores: a reanalysis of two clinical trials of postoperative pain. J Pain 2003;4:407-14.

19. Verma A, Jiang CY, Betts TR, Chen J, Deisenhofer I, Mantovan R, et al. Approaches to catheter ablation for persistent atrial fibrilla- tion. N Engl J Med 2015;372:1812-22.

20. Cardoso R, Knijnik L, Bhonsale A, Miller J, Nasi G, Rivera M, et al. An updated meta-analysis of novel oral anticoagulants versus vitamin K antagonists for uninterrupted anticoagulation in atrial fibrillation catheter ablation. Heart Rhythm 2018;15:107-15.

21. Aldhoon B, Wichterle D, Peichl P, Čihák R, Kautzner J. Complications of catheter ablation for atrial fibrillation in a high-volume centre with the use of intracardiac echocardiography. Europace 2013;15:24-32.

22. Deshmukh A, Patel NJ, Pant S, Shah N, Chothani A, Mehta K, et al. In-hospital complications associated with catheter ablation of atrial fibrillation in the United States between 2000 and 2010: analysis of 93801 procedures. Circulation 2013;128:2104-12.

23. Gupta A, Perera T, Ganesan A, Sullivan T, Lau DH, Roberts-Thomson $\mathrm{KC}$, et al. Complications of catheter ablation of atrial fibrillation: a systematic review. Circ Arrhythm Electrophysiol 2013;6: 1082-8.

24. Ferguson JD, Helms A, Mangrum JM, Mahapatra S, Mason P, Bilchick K, et al. Catheter ablation of atrial fibrillation without fluoroscopy using intracardiac echocardiography and electroanatomic mapping. Circ Arrhythm Electrophysiol 2009;2:611-9.

25. Mun HS, Joung B, Shim J, Hwang HJ, Kim JY, Lee MH, et al. Does additional linear ablation after circumferential pulmonary vein isolation improve clinical outcome in patients with paroxysmal atrial fibrillation? Prospective randomised study. Heart 2012;98: 480-4.

26. Su W, Kowal R, Kowalski M, Metzner A, Svinarich JT, Wheelan K, et al. Best practice guide for cryoballoon ablation in atrial fibrillation: the compilation experience of more than 3000 procedures. Heart Rhythm 2015;12:1658-66.

27. Ezzat VA, Chew A, McCready JW, Lambiase PD, Chow AW, Lowe $\mathrm{MD}$, et al. Catheter ablation of atrial fibrillation-patient satisfaction from a single-center UK experience. J Interv Card Electrophysiol 2013;37:291-303.

28. Robbins IM, Colvin EV, Doyle TP, Kemp WE, Loyd JE, McMahon WS, et al. Pulmonary vein stenosis after catheter ablation of atrial fibrillation. Circulation 1998;98:1769-75.

29. Kuwahara T, Takahashi A, Takahashi Y, Kobori A, Miyazaki S, Takei A, et al. Clinical characteristics of massive air embolism complicating left atrial ablation of atrial fibrillation: lessons from five cases. Europace 2012;14:204-8. 